Check for updates

Cite this: Mater. Adv., 2022, 3, 173

Received 25th September 2021, Accepted 22nd November 2021

DOI: $10.1039 / \mathrm{d} 1 \mathrm{ma} 00885 \mathrm{~d}$

rsc.li/materials-advances

\section{Liquid metals as soft electromechanical actuators}

\author{
Tim Cole (D) and Shi-Yang Tang (D)*
}

\begin{abstract}
Leveraging the unique properties of liquids, such as surface tension, capillary action, reconfigurability, nearly unlimited stretchability, and viscosity has enabled the development of a wide range of soft actuators, presenting vast potential to revolutionise wearable healthcare devices, manufacturing, reconfigurable electronics, and robotics. Gallium (Ga) based liquid metals (GaLMs) are a remarkable family of functional fluidic materials that can actuate electrically for realising electromechanical functions. Such actuators are simple, highly responsive, highly controllable, and reversible, which has led to the creation of useful devices such as reconfigurable antennas, artificial muscles, electrical switches, and soft robots, just to name a few. Herein, this review succinctly and critically summarises recent advances in research on using GaLMs as electromechanical actuators. First, the properties of GaLMs are introduced, and then the methods for their electrical actuation and the applications thereof are discussed. Finally, an outlook is offered, highlighting the research challenges faced by liquid metal electromechanical actuators in order to develop into commercial devices.
\end{abstract}

\section{Introduction}

Transformable soft actuators constituting highly deformable materials with low moduli, such as polymers, gels, and fluids play an important role in actuating robotic systems, particularly for those with soft moving bodies. ${ }^{1,2}$ These actuators generate

Department of Electronic, Electrical and Systems Engineering, University of Birmingham, Edgbaston, Birmingham, B15 2TT, UK. E-mail: S.Tang@bham.ac.uk motions and forces upon the application of external stimuli, such as electric/magnetic fields, ${ }^{3-8}$ pressure/temperature changes, ${ }^{9-12}$ light, ${ }^{13-15}$ and chemicals. ${ }^{16,17}$ Unlike their rigid counterparts, soft actuators can easily deform and adapt to dynamic conditions in changing environments.

Electromechanical soft actuators transduce electrical energy into mechanical motions or deformation. Among all stimuli, electrical signals have numerous advantages; for example, they allow for easy control of magnitude, frequency, and phase.

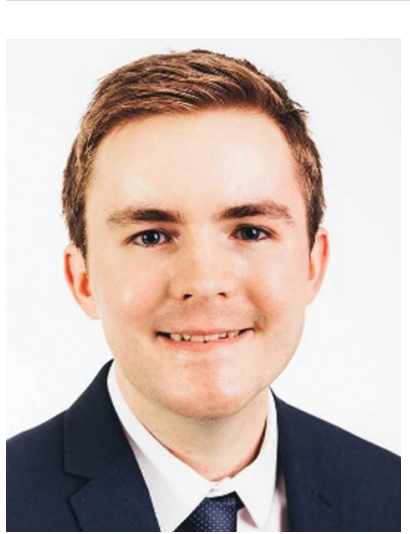

Tim Cole
Tim Cole obtained his BSc in physics from the University of Manchester in 2016 and his MSc in advanced mechanical engineering from the University of Birmingham in 2020. He is currently a PhD student at the University of Birmingham, UK. His research interests include soft robotics and liquid metal embedded composites.

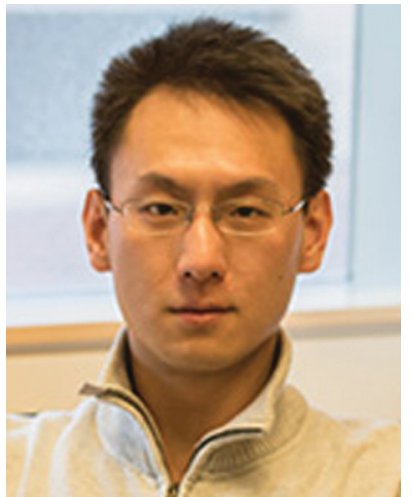

Shi-Yang Tang
Dr Shiyang Tang currently is a Lecturer (Assistant Professor) in the Department of Electronic, Electrical and Systems Engineering at the University of Birmingham, UK. He received his BEng (1st class honours) in Electrical Engineering and $P h D$ in Microelectromechanical Systems (MEMS) from the RMIT University, Australia, in 2012 and 2015, respectively. He was the recipient of the Discovery Early Career Researcher Award from the Australian Research Council, and the Vice-Chancellor's Postdoctoral Research Fellow from the University of Wollongong, Australia. Dr Tang's research interests include developing microfluidic platforms for biomedical studies and liquid metal enabled micro-/nanoscale platforms. He has published more than 85 journal papers. 
Additionally, components for generating electrical stimuli are compatible with conventional electronics and therefore, could be readily integrated with and powered by batteries. The design, synthesis, and integration of non-ionic (e.g., dielectric elastomers, electrostrictive polymers, liquid crystal elastomers, etc. $)^{4,13,18-20}$ and ionic (e.g., ionic conducting polymers and their composites, ionic gels, etc. $)^{21-23}$ electro-responsive polymers, as well as fluids that can change viscosity, surface tension, and pressure upon electrical stimulation ${ }^{5,24-27}$ have been extensively explored to make soft actuators over the past decade.

Liquid enabled soft actuators are able to make use of the inherent advantages of fluidic systems. For example, fluids can deform freely without mechanical constraints to movement and are naturally self-healable. Thus, incorporating electroresponsive fluids in solid materials, or using fluids themselves as actuators minimises mechanical fatigue and heals electrical breakdown. ${ }^{3}$ Moreover, interfacial tension of fluids can be readily tuned electrically using the electrowetting effect, making fluid actuators an attractive alternative in small-scale robotic systems. ${ }^{2,25}$

GaLMs provide many unique properties that make them particularly suited for usage in soft actuators. For instance, GaLMs have the highest surface tension (>600 $\mathrm{mN} \mathrm{m}^{-1}$ ) among all liquids and have a negligible vapour pressure even at a high temperature $\left(>500{ }^{\circ} \mathrm{C}\right) .{ }^{28}$ They are also immiscible in aqueous and organic fluids. More importantly, their metallic properties enable many extraordinary effects that cannot be reproduced using conventional fluids, such as electrochemical oxidation/reduction, continuous electrowetting, and the induction of the Lorentz force. These effects have enabled unparalleled actuation methods that have been harnessed for making innovative electromechanical soft actuators, and eventually lead to the construction of more complex systems for various applications (Fig. 1).

This review seeks to summarise and highlight the fundamental principles and applications of GaLM enabled electromechanical actuators. We will first briefly discuss the unique properties offered by GaLMs that can be harnessed for realising electromechanical effects. After this, the different electronically controlled actuation methods are described, alongside examples of its various uses in robotic, electronic, and microfluidic systems. Finally, we offer a perspective on the opportunities and challenges for the future development of such GaLM-based electromechanical actuators.

\section{Liquid metal enabled electromechanical actuators}

\subsection{Brief summary of the properties of gallium-based liquid metals}

GaLMs includes alloys such as eutectic gallium indium (EGaIn, $75 \mathrm{wt} \% \mathrm{Ga}, 25 \mathrm{wt} \% \mathrm{In})^{29}$ and Galinstan (68\% wt\% Ga, $22 \mathrm{wt} \%$ In, $10 \mathrm{wt} \% \mathrm{Sn}) .{ }^{28}$ Pure Ga has a melting point of $29.8{ }^{\circ} \mathrm{C},{ }^{29}$ slightly above room temperature, whereas EGaIn and Galinstan have melting points of $15.7{ }^{\circ} \mathrm{C}$ and $\sim 11{ }^{\circ} \mathrm{C}$ respectively. ${ }^{29,30} \mathrm{In}$ contrast to mercury, GaLMs have a very low toxicity. ${ }^{31}$ They also have a negligible vapor pressure, ${ }^{28,29}$ meaning there is no danger of accidental inhalation. Another feature of GaLMs is its oxide layer 'skin' which quickly forms in air with a thickness of between $0.7-3 \mathrm{~nm} .{ }^{32}$ Oxide formation occurs even at very low concentrations of oxygen ( $\sim$ few ppm). ${ }^{32}$ The oxide layer reduces the surface tension from $>600 \mathrm{mN} \mathrm{m}^{-1}$ for bare GaLMs $^{33,34}$ to $\sim 350 \mathrm{mN} \mathrm{m}{ }^{-1}$. $^{35}$ Interfacial tension is also reduced when GaLM is immersed in electrolyte (down to $415 \mathrm{mN} \mathrm{m}^{-1}$ in $1 \mathrm{M}$ sodium hydroxide, $\left.\mathrm{NaOH}\right)^{36}$ due to electrical double layer formation. The oxide layer can be removed chemically by using acid or base, or electrochemically. ${ }^{37,38}$ With the oxide layer present, the LM has increased adhesion, which can be used for patterning the LM. ${ }^{39}$ However, the stabilizing effect and adhesion of the oxide also stops the bulk metal from flowing, so in some circumstances the oxide layer has to be continuously removed, or a surface coating applied to prevent the oxide sticking: ${ }^{40}$ GaLMs also readily alloy with some metals, such as copper, ${ }^{41}$ and aggressively corrodes some aluminium alloys. ${ }^{42}$ Other metals it has been shown to be non-corrosive towards however. ${ }^{42}$ A comprehensive review on the properties of GaLMs can be found elsewhere. ${ }^{32,43-45}$

The high conductivity and fluidity of GaLM, and its oxide layer mean that it can be actuated using various electrically based means. As the focus of this review is on electrically based actuation, other ways to actuate GaLM (such as by utilizing the redox reaction between GaLM and aluminium in $\mathrm{NaOH}$

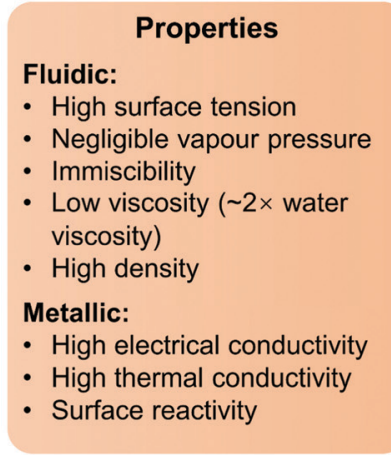

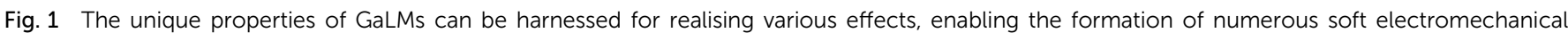
actuators and eventually lead to applications in more complex systems. 
solution $)^{46}$ will not be discussed. See two recent review papers for more information on these other actuation regimes. ${ }^{47,48}$ In this review, the focus is on GaLMs. Both of the terms 'LM' and 'GaLM' are used in the review but should be understood to refer to GaLMs only. See elsewhere ${ }^{49}$ for a review that discusses actuation by interfacial tension modulation of both mercury and GaLMs.

\subsection{Interfacial tension modulation by electrochemical oxidation and reduction}

GaLMs exhibit a huge change in interfacial tension from $>400 \mathrm{mN} \mathrm{m}^{-1}$ to $\sim 0 \mathrm{mN} \mathrm{m}^{-1}$ under certain conditions. ${ }^{38}$ This change is fast $(\ll 1 \mathrm{~s})$, reversible, and only requires a small voltage $(\sim 1 \mathrm{~V})$. The interfacial tension reduction is achieved by electrochemically oxidising the LM droplet while in electrolyte, making an oxide layer grow. If the electrolyte used is an acid or base that removes the oxide layer, then the oxide is continually removed as it is formed. This means the LM has no impedance to its spreading, and with such a low interfacial tension, it flattens and forms random shapes (Fig. 2A). ${ }^{50}$ The interfacial tension can be increased back to its maximum value of $>400 \mathrm{mN} \mathrm{m}^{-1}$ by removing the oxidative potential if in acid or base, or by applying a reductive potential. This makes the droplet reform its spherical shape. The interfacial tension change arises from the oxide layer acting as a surfactant and compressive stresses resulting from oxidation. ${ }^{50,51}$ The speed and repeatability of this effect have been showcased by using it to make droplets that jump over $5 \mathrm{~mm}$ in electrolyte (Fig. 2B), ${ }^{52}$ and a beating heart gallium droplet that is capable of 610 beats per minute. ${ }^{53}$

The shape of LM in 2D can be controlled electrochemically using oxidation and the Marangoni effect. ${ }^{54}$ The Marangoni effect is when liquid flows from regions of low interfacial tension to regions of high interfacial tension. To achieve the shape control, a LM droplet was placed in a round dish with an anode touching it, immersed in $\mathrm{NaOH}$ electrolyte, and with cathodes at the edge of the dish. By applying a potential, oxide forms preferentially where the LM side is facing a cathode. This reduces its interfacial tension locally, and results in Marangoni flow towards where the LM has a higher interfacial tension at the centre of the dish. This makes the LM spread towards the cathodes (Fig. 2C). The LM could be spread to up to three different cathodes. The minimum angle between two protrusions was $30^{\circ}$. Using a feedback control system, the LM stayed in position for up to $12 \mathrm{~s}$.
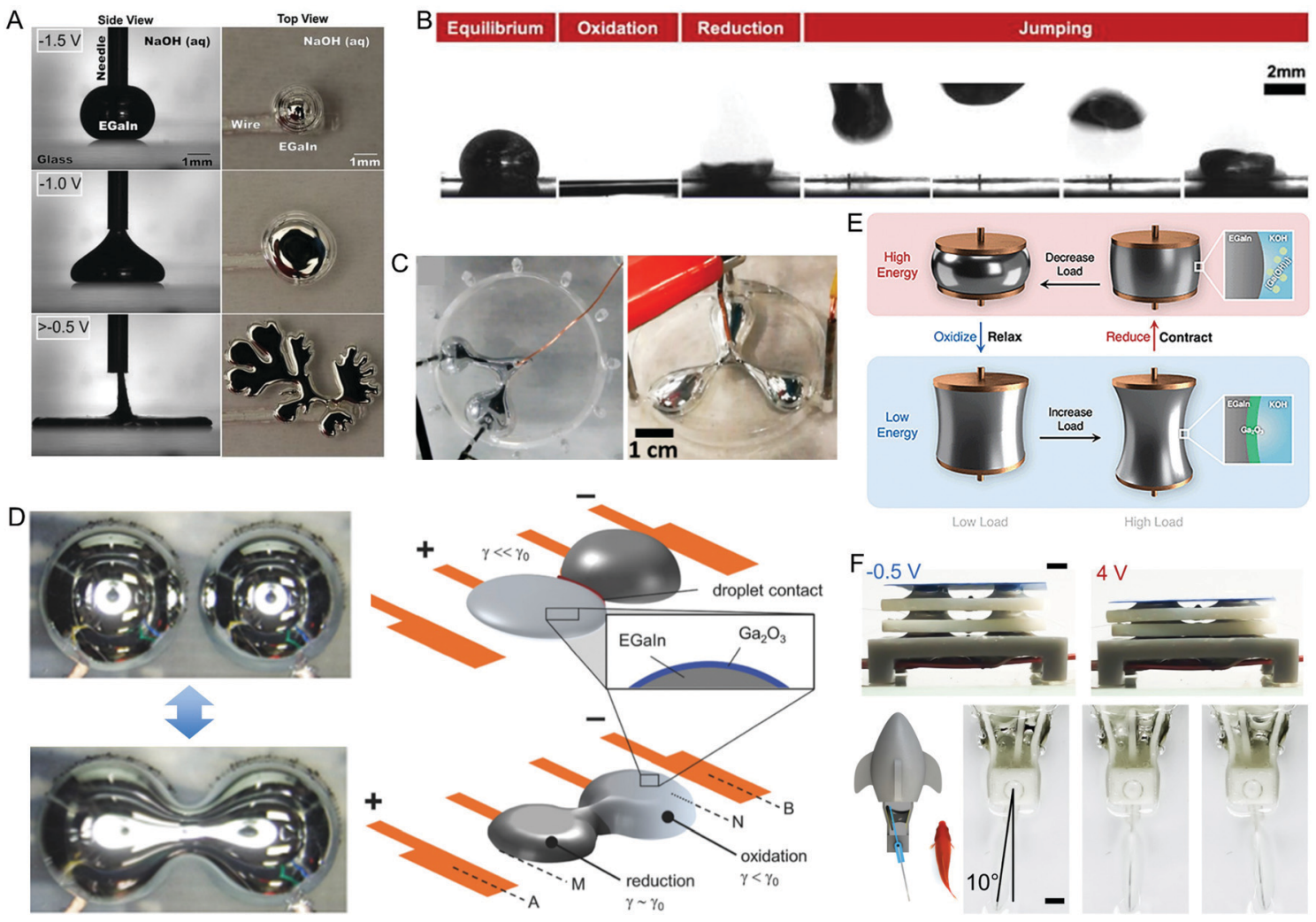

Fig. 2 Actuators enabled by oxidation and reduction of LM. (A) Oxidation of LM immersed in NaOH solution causes its interfacial tension to reduce significantly, resulting in flattening and spreading of the LM. Reproduced with permission. ${ }^{38}$ Copyright 2014, National Academy of Sciences. (B) A LM droplet jumps over $5 \mathrm{~mm}$ high due to oxidation and reduction. Reproduced with permission. ${ }^{52}$ Copyright 2021, American Institute of Physics. (C) LM shape control using electrochemistry. Reproduced with permission. ${ }^{54}$ Copyright 2019, MDPI. (D) Merging and separation of LM used as an electrical switch. Reproduced with permission. ${ }^{55}$ Copyright 2017, Wiley-VCH. (E) A LM artificial muscle with copper pads on either side to pin the LM. Contraction and relaxation of the LM artificial muscle can be achieved upon oxidation or reduction. Reproduced with permission. ${ }^{57}$ Copyright 2021 , Royal Society of Chemistry. (F) Actuation of a 3-layer stack LM artificial muscle and using a LM artificial muscle to swing the caudal fin of an untethered bionic robotic fish. Scale bars are $2 \mathrm{~mm}$. Reproduced with permission. ${ }^{56}$ Copyright 2021, Wiley-VCH. 
A switch that makes use of oxidation and reduction to coalesce and split LM droplets has also been made. ${ }^{55}$ The switch consists of electrolyte encapsulating two LM droplets wetted on two copper electrodes, with two additional outer electrodes on either side. The LM preferentially wets the copper by alloying, ${ }^{41}$ preventing the LM moving off it. When the switch is off, the droplets are separate. To turn the switch on, an oxidative potential is applied to one droplet and a reductive potential to the outer electrode on the far side. This causes one droplet to oxidise and spread out, merging with the second droplet (Fig. 2D). To split the droplets, a positive potential is applied to one outer electrode and a negative potential is applied to the other outer electrode. This causes one side of the merged droplet to oxidise, and one side to be reduced, which makes the droplet unstable and splits.

Soft artificial muscles have been made that make use of the change in surface tension of LM. ${ }^{56-58}$ As LM preferentially wets copper by alloying, two copper pads can be used to hold the droplet in place for actuation (Fig. 2E). The droplet is then immersed in an electrolyte, with a periodically oscillating potential applied to it. The electrolyte is usually a caustic acid or base to increase the speed at which the oxide layer is removed, although an alternative electrolyte (such as sodium chloride solution) has been shown to work if a reductive potential removes the oxide layer. ${ }^{56}$ With the LM droplet pinned from above and below, the force generated can result in either pushing or pulling of the pads, depending on the configuration. ${ }^{56}$ Artificial muscle droplets can be used in parallel to increase the force or in series to increase the stroke (Fig. 2F). The artificial muscle droplets are unstable if the distance between the pads becomes too great, as this causes the LM droplet to be pulled apart during actuation. Maximum distance between the pads is $\sim 2-4 \mathrm{~mm}$ for a $1 \mathrm{~mm}$ radius $\mathrm{LM}$ droplet muscle, depending on its oxidation/reduction state and load force. ${ }^{57}$ Another issue is that electrolysis of the electrolyte during electrochemical reactions causes hydrogen to be formed as an unwanted by-product. The gas generation can degrade the performance of the actuator. If the voltage used is too high $(\sim 8 \mathrm{~V})$ the amount of bubbles generated can block the actuation. ${ }^{56}$ Gas build-up would be a major issue in sealed contractile units, and requires release valves to be used, or an alternative electrolyte that does not create a gaseous by-product.
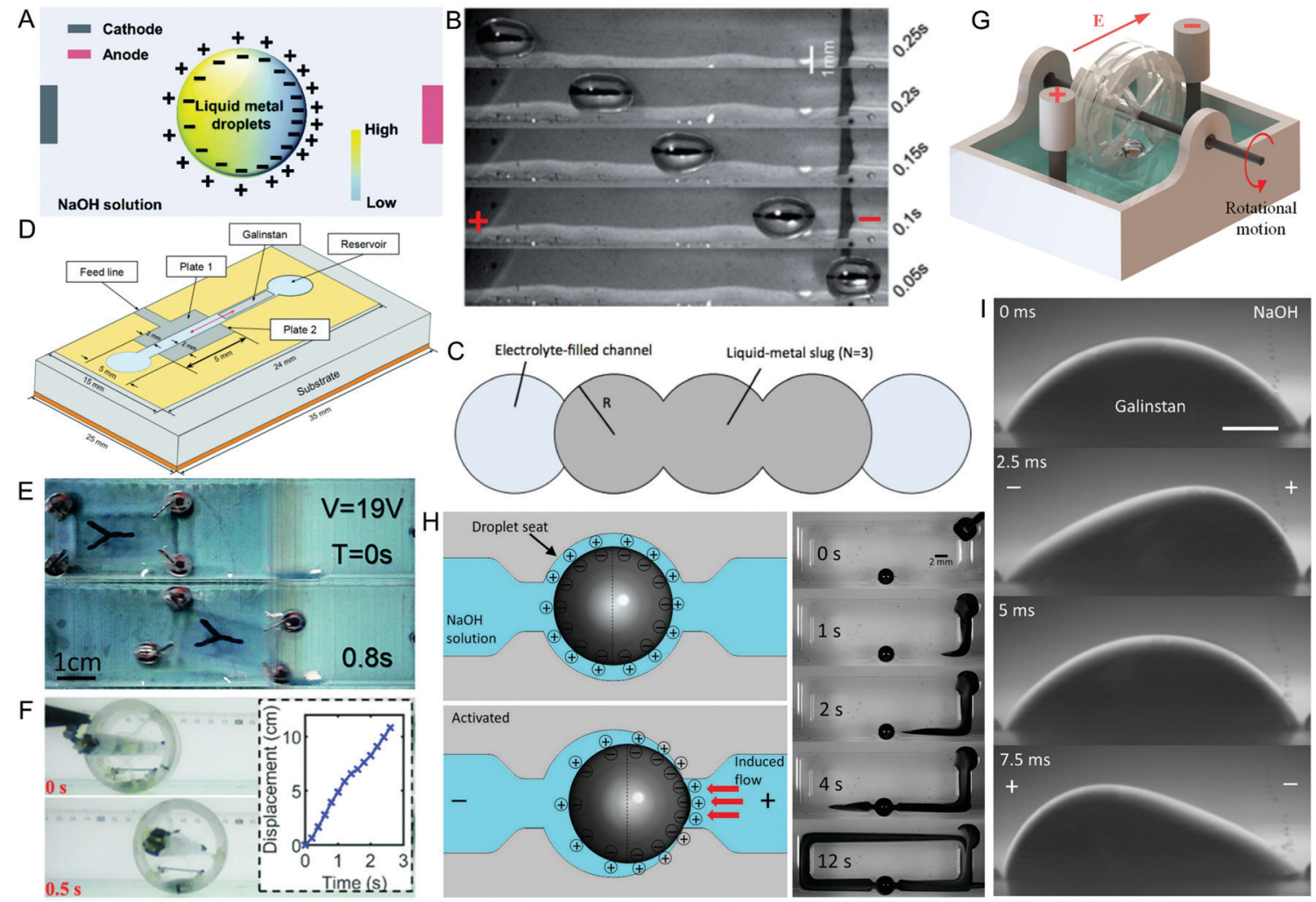

Fig. 3 Actuators enabled by the CEW effect of LM. (A) Distribution of the EDL on LM immersed in NaOH solution with an applied potential across it. Reproduced with permission. ${ }^{66}$ Copyright 2021, Royal Society of Chemistry. (B) LM moving in a channel, from the cathode towards the anode, due to CEW. Adapted with permission. ${ }^{65}$ Copyright 2013, Royal Society of Chemistry. (C) Shape of a channel to cause minimum energy states of LM, keeping it in the desired position. Reproduced with permission. ${ }^{69}$ Copyright 2014, IEEE. (D) Diagram of a variable parallel-plate capacitor. Reproduced with permission. ${ }^{70}$ Copyright 2021, IEEE. (E) A small vehicle with LM 'wheels'. Reproduced with permission. ${ }^{71}$ Copyright 2016, Royal Society of Chemistry. (F) Side view of a wheeled robot driven by a change in centre of gravity moving outside of electrolyte. Reproduced with permission. ${ }^{72}$ Copyright 2018 , Wiley-VCH. (G) A universal mechanical module that is converts the flow caused by LM CEW into mechanical work. Reproduced with permission. ${ }^{73}$ Copyright 2021, Royal Society of Chemistry. (H) A LM pump. Reproduced with permission. ${ }^{74}$ Copyright 2014, National Academy of Sciences. (I) An AC potential applied across LM causes it to oscillate. Reproduced with permission. ${ }^{75}$ Copyright 2014 , Wiley-VCH. 
The artificial muscles are able to actuate with strains up to $87 \%$, with negligible response time. ${ }^{56}$ Voltages typically used for actuation were from $4 \mathrm{~V}$ to $-0.5 \mathrm{~V}$. The artificial muscle was able to actuate repeatedly at $0.5 \mathrm{~Hz}$ for 2 hours with no loss of performance. LM artificial muscle was used to make an untethered swimming fish (Fig. 2F), a bimodal display, cargo carrier and reconfigurable optical reflector. ${ }^{56}$ The work density of such LM muscles created with $1 \mathrm{~mm}$ diameter droplets is $\sim 100 \mathrm{~kJ} \mathrm{~m}^{-3} \cdot{ }^{57}$ Reducing the size of the droplets will result in a greater energy density due to an increase in the surface area to volume ratio. Note that interfacial tension scales linearly with length $(L)$, whereas forces such as electrostatic and magnetic scale as $L^{2}$ and $L^{3}$ respectively. ${ }^{2}$ This means that interfacial tension forces dominate at small scales. A droplet size of $2 \mu \mathrm{m}$ will result in a theoretical energy density of $10^{3} \mathrm{kJm}^{-3}$, greater than dielectric elastomers and shape memory alloys. ${ }^{57}$

\subsection{Continuous electrowetting}

When a LM droplet is immersed in an electrolyte, it gains a net surface charge. For example, when in $\mathrm{NaOH}$ solution, chemical reactions result in formation of $\left[\mathrm{Ga}(\mathrm{OH})_{4}\right]^{-}$on the LM surface. An electrical double layer (EDL) is then formed around the droplet as oppositely charged ions in the electrolyte are attracted towards it (Fig. 3A). ${ }^{59}$ The interfacial tension varies with the potential across it. The Young-Lippman equation describes the variation of the interfacial tension, $\gamma$ :

$$
\gamma=\gamma_{0}-\frac{1}{2} C V_{\mathrm{EDL}}^{2}
$$

where $\gamma_{0}$ is the maximum interfacial tension at the point of zero charge, $C$ is the capacitance per unit area of the EDL, and $V_{\mathrm{EDL}}$ is the voltage across the EDL. ${ }^{60}$ When a potential is applied across a LM droplet in an electrolyte, the low electrical conductivity of the electrolyte means that there is a potential drop along the channel. The LM, however, has a high conductivity, so its potential can be regarded as the same at all points on the droplet. Therefore, the potential difference across the EDL is higher at one side of the droplet than the other. This results in a non-uniform interfacial tension. With no external applied potential, the interfacial tension everywhere on the droplet is still uniform.

When there is an interfacial tension gradient across the LM droplet, this results in the generation of Marangoni flows along the surface to drive the droplet towards the anode (Fig. 3A and B). It also results in flow of electrolyte from the lower interfacial tension side of the droplet to the higher interfacial tension side. This type of LM actuation is known as continuous electrowetting (CEW). CEW offers a large degree of control over the movement of the LM droplet. LM can be controlled in two dimensions, ${ }^{61}$ and even made to travel up a slope. ${ }^{62}$

Accurate manipulation of multiple LM droplets in 2D has been achieved using infrared lasers to selectively trigger phototransistors. ${ }^{63}$ The experimental set up used contains a grounded graphite electrode, which surrounds a circuit board immersed in $\mathrm{NaOH}$ solution, with a phototransistor and copper electrode array on it. A transparent epoxy is coated on the circuit board, with only the tips of the electrodes exposed, meaning the LM droplets are able to move freely. When a laser is shone onto a phototransistor, the appropriate copper electrode is activated, and a LM droplet is actuated towards it by CEW. This can be used to control the position of multiple LM droplets concurrently. It can also be used to merge droplets by moving them at speed towards one another. If the laser is left on a phototransistor while the LM is situated on its electrode, the LM is oxidised, flattens and spreads. Removing the laser stops the oxidation, and the $\mathrm{NaOH}$ solution causes the oxide to be removed, beading the LM back up to a spherical shape. The LM can be split into two droplets during this process if it spread enough during its oxidation.

The electrolyte used affects the actuation of the LM. For example, using a hydrochloric acid $(\mathrm{HCl})$ solution rather than $\mathrm{NaOH}$ results in the LM surface becoming positively charged ${ }^{64}$ (it is negatively charged in $\mathrm{NaOH}$ solution). An EDL then forms which has a reversed polarity compared with that made in $\mathrm{NaOH}$ solution. This causes the LM to travel to the cathode rather than the anode. The LM also moves slower and needs a greater potential however $(25 \mathrm{~V}$ minimum for actuation compared with $2 \mathrm{~V}){ }^{65}$ The worse performance is due to the low surface activity of chloride ions. ${ }^{36}$ Using an acidified (0.1 M $\mathrm{HCl}$ ) potassium iodide (KI) solution generates a higher surface charge density on the EDL due to iodide ion adsorption. ${ }^{36}$ This gives improved electrical actuation in an acidic electrolyte, and it even exhibits better performance than when using $\mathrm{NaOH}$ electrolyte. The actuation required a lower voltage ( $4 \mathrm{~V}$ rather than $9 \mathrm{~V}$ ) and droplets moved faster. ${ }^{36}$

When immersed in $\mathrm{NaOH}$ solution, LM droplet speed initially increases with greater potential applied. ${ }^{66}$ Once a maximum droplet speed is reached however, an increased potential does not result in greater interfacial tension difference on the LM droplet, and hence its speed is not increased. If the potential applied is large enough, it can cause oxidation of LM, making its interfacial tension gradient in the opposite direction, causing the droplet to travel towards the cathode rather than the anode. $^{66}$

A surface coating applied to the LM can also affect its actuation behaviours. Micro- or nanoparticles can coat the surface of GaLM droplets to form LM marbles. ${ }^{67}$ For LM marbles with semiconductive nanoparticle coatings, a n-type nanomaterial coating (e.g., tungsten trioxide, $\mathrm{WO}_{3}$ ) induces actuation in a way similar to that of bare LM droplets in $\mathrm{NaOH}$ solution - the marble moves towards the anode upon the application of an external potential gradient. ${ }^{65}$ However, a p-type nanomaterial coating (e.g., cupric oxide, $\mathrm{CuO}$ ) induces more complex actuation behaviours - the marble elongates and actuates towards the cathode while the nanoparticles migrate to form a dense cluster tail. ${ }^{68}$ The tail eventually falls off and then the LM droplet abruptly changes the direction of actuation towards the anode.

Although the voltages used for LM CEW actuation are relatively low ( $\sim$ few volts usually), the potential difference is still large enough to for electrolysis to occur $(>1.2 \mathrm{~V}$ required for $\mathrm{H}_{2}$ generation). However, bubble generation has been shown to have only a very slight impact on the actuation of the LM. ${ }^{65}$ 
This was achieved by comparing the actuation of stainless steel beads and LM in electrolyte solution with a potential difference across them. The bead actuation was caused only by bubble generation, whereas LM actuation was caused by both bubble generation and the surface tension gradient. The beads moved very slowly in $\mathrm{HCl}$ solution $\left(<0.2 \mathrm{~mm} \mathrm{~s}^{-1}\right)$, and didn't move at all in $\mathrm{NaOH}$ solution. In contrast, LM actuation was much faster - up to $67 \mathrm{~mm} \mathrm{~s}^{-1}$ in $\mathrm{NaOH}$ solution and $33 \mathrm{~mm} \mathrm{~s}^{-1}$ in $\mathrm{HCl}$ solution. This shows that the surface tension gradient has a much greater effect on LM actuation than bubble generation.

The high conductivity and fluidity of LM means it is particularly useful in making reconfigurable antennas. ${ }^{76}$ The LM can be moved to different positions to change the properties of the antenna. Accurate positioning of the LM is important for radio frequency applications; however, this is difficult to achieve when actuating LM using CEW due to the inertia of the LM and low friction between LM and the channel. ${ }^{69}$ Also, the motion of the LM can create a pressure differential between the electrolyte at either side of it. After actuation, the pressure differential could cause the LM to move from its desired position. ${ }^{69}$ One method to improve accuracy of positioning of the LM is to shape the channel to create minimum energy states for the LM droplet. ${ }^{69}$ An example of this is a series of rounded indentations (Fig. 3C). The high surface tension of LM means that it will try to minimise its surface area. Therefore, after actuation, it will stay within a set position. This results in a discrete number of positions of the droplet and corresponding radio frequency properties.

A radio frequency shunt switch has also been made which makes use of CEW of LM. ${ }^{77}$ For the switch, a signal line is placed across a channel, and LM is actuated into a position over the signal line to complete the connection to ground. If no LM is above the signal line, then the connection to ground is removed. The use of electrolyte in radio frequency devices can introduce unwanted losses, however. In this case, when electrolyte is above the signal line, it absorbs the radio frequency signal. In order to use CEW while reducing the losses due to electrolyte, the switch design used a bubble trapped in the channel, and capillary troughs to carry the electrolyte to either side of the main channel. The capillary troughs gave a continuous path in the electrolyte for CEW to function. The troughs were smaller than the channel containing the LM, preventing the LM from moving into the troughs as this would increase its surface area. A bubble was positioned between two LM droplets, it stayed in position due to capillary action of the electrolyte preferentially bringing the electrolyte into the trough rather than the bubble. This design reduced the losses caused by the electrolyte.

CEW of LM can also be used for tuneable capacitors. ${ }^{70}$ The tuneable parallel-plate capacitor comprises a copper bottom layer with dielectric above it, and a top conductor made of two stainless steel plates, with a channel placed between the plates (Fig. 3D). The channel is filled with electrolyte and a LM slug. Actuating the LM slug by CEW to bridge the gap between the two stainless steel plates causes the effective area of the plates to be increased, resulting in a higher capacitance. The capacitance varied from $9.76 \mathrm{pF}$ with the LM slug not contacting the plates to $10.34 \mathrm{pF}$ with the slug fully between the plates.

LM droplets can be used to propel vehicles by using the droplets as soft 'wheels' in electrolyte (Fig. 3E). ${ }^{71,78}$ By building a platform around the droplets, cargo can be carried, and the droplets are steered by CEW to a specified location. Alternatively, the LM droplet itself can be used as the vehicle. ${ }^{79}$ For example, as LM preferentially wets copper, LM was used to encapsulate a hollow copper-coated sphere. The hollow sphere contained three cabins - the driving, counterweight, and cargo cabins. The driving cabin contained magnetic particles to aid in moving the droplet in addition to using CEW. The counterweight was used to keep the correct position of the sphere within the droplet. The cargo cabin was filled with the payload, and was sealed with wax, which could be melted with a laser. The droplet was successfully actuated using CEW and magnets before unloading its payload.

LM soft robots are interesting but have a major drawback they are only able to move in an electrolyte, which limits their real-world applications. It is possible to use LM to actuate robots outside of an electrolyte, however. This has been achieved by encasing a channel with a LM droplet, electrolyte, electrodes and a battery within an untethered wheeled robot. $^{72}$ The LM position changes depending on the potential applied, which in turn causes the centre of gravity of the wheel to alter, making the wheeled robot move (Fig. 3F).

A similar, alternative approach is to create a LM universal mechanical module, which is inspired by a water wheel (Fig. 3G). ${ }^{73}$ CEW of LM drives the spinning of the wheel, which is then used to generate rotational motion outside of the solution. Aluminium flakes were also added to the LM, which created a greater negative charge on the LM due to the galvanic reaction between aluminium, EGaIn and $\mathrm{NaOH}$. The greater charge is then utilised to generate a stronger Marangoni flow with the applied potential. This in turn creates a larger force from the module.

Another device that uses LM to do mechanical work is a LM motor. ${ }^{80}$ The motor contains a rotor with multiple LM actuating units. ${ }^{80}$ Each of the actuating units contained a LM droplet immersed in $\mathrm{NaOH}$ electrolyte, controlled with a pair of electrodes. The actuating units work together to generate a continuous output torque that is greater than that made by a single LM droplet. LM was also used as the electric brush between the rotor and stator, making use of its high conductivity and low viscosity. This reduced friction and prevents wear and sparks. The motor was used to successfully drive an untethered vehicle and boat.

When a LM droplet is trapped within an electrolyte filled chamber with an applied electric potential across it, it functions as a pump. ${ }^{74}$ The surface tension gradient causes Marangoni flow around the droplet, but as the droplet it trapped, it cannot move out of the chamber. Therefore, the electrolyte is pumped by the droplet (Fig. 3H). The applied potential may cause an oxide layer to form on the more anodic pole of the droplet after a few seconds. This would then stop the pumping due to the reduction in the surface tension on that side (as that was the side with the highest surface tension previously). Oxidation can be 
prevented by using an AC potential difference with a DC offset. ${ }^{74}$ The optimum frequency $(\sim 200 \mathrm{~Hz})$ ensures surface charges can be accumulated and released, preventing oxidation and allowing pumping to continue. Too low of a frequency results in gradual oxidation. Too high of a frequency means that the ions in the EDL do not have time to redistribute, reducing the pressure difference across the droplet and lowering the pumping rate. Under optimum conditions, the LM pump exhibited a high flow rate with low power consumption. It was shown to work with a range of electrolytes including solutions of $\mathrm{NaOH}, \mathrm{NaCl}$, and phosphate buffered saline. It could also pump a liquid made by mixing glycerol and deionised water, which had a viscosity of $0.209 \mathrm{~N} \mathrm{~s} \mathrm{~m}^{-2}$ ( $\sim 230$ times water viscosity). LM pumps have been used as cooling systems, ${ }^{81}$ and have also been shown to be able to pump an ionic liquid. ${ }^{82}$ Using ionic liquid offers an advantage over using $\mathrm{NaOH}$ as electrolyte due to an increased interfacial ion adsorption, which results in a higher pumping rate.

In addition to pumping of liquid, mixing is also important for flows at low Reynolds numbers. Mixing can be achieved by applying an AC electric potential in electrolyte to a LM droplet wetting on a copper pad, causing the Marangoni flow generated at the surface of the LM to oscillate back and forth (Fig. 3I). ${ }^{75}$ The oscillating potential causes the interfacial tension on different parts of the surface to vary rapidly with time, and LM wetted onto the copper pad prevents the bottom layer of the droplet moving. Mixing can also be achieved without the use of a copper pad. ${ }^{83}$ By using a DC biased AC potential with a greater potential variance, this induces chaotic advection between the neighbouring laminar flow.

\subsection{Electrocapillarity}

The flow of LM in capillaries can be achieved by using electrochemical and electrowetting techniques- both of which will be elaborated on here.

Electrochemically controlled capillarity (or 'recapillarity') involves removing the oxide layer of GaLM, causing LM to retreat from a channel (Fig. 4A). ${ }^{84}$ This prevents the oxide layer from sticking to the walls of the channel (which can occur if the LM is pumped out). A non-caustic electrolyte such as sodium fluoride ( $\mathrm{NaF}$ ) is used for electrochemically controlled capillarity, as it does not remove the oxide without a reducing potential. Applying a reducing potential to the LM causes a current to flow and the LM to move out of the channel due to removal of the oxide and its increase in interfacial tension. Removing the potential stops the current and the LM flow, because oxide that formed on the sidewalls of the channel needs to be removed for the LM to flow out. Speeds of up to $0.3 \mathrm{~ms}^{-1}$ have been achieved using a high concentration of electrolyte ( $1 \mathrm{M} \mathrm{NaF}$ ). Localised reduction of planar GaLM is also able to 'write' by removing LM near an electrode (Fig. $4 \mathrm{~B}$ and C). ${ }^{85}$

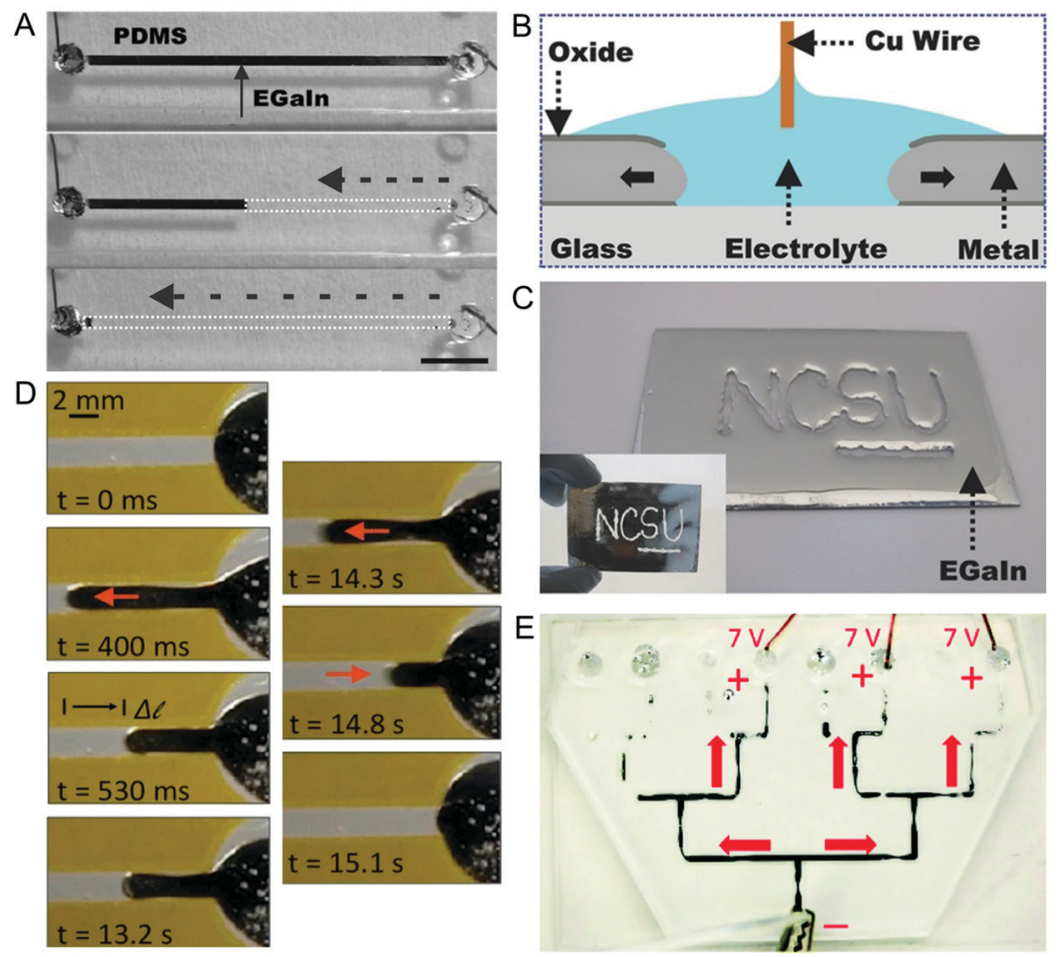

Fig. 4 Actuators enabled by electrocapillarity flow of LM. (A) Recapillarity-reduction of LM in a channel causes it to flow out. Reproduced with permission. ${ }^{84}$ Copyright 2015, Wiley-VCH. (B) In-plane recapillarity schematic. (C) Writing using in-plane recapillarity. Reproduced with permission. ${ }^{85}$ Copyright 2016, Wiley-VCH. (D) Electrocapillarity causes LM to flow out of a reservoir. The potential is then flipped and the LM is oxidised to keep it in place. After this, the potential is reversed again, reducing the oxide and the LM retreats to the reservoir. Reproduced with permission. ${ }^{59}$ Copyright 2015 , Springer. (E) Directing LM flow to three end points in branching channels. Reproduced with permission. ${ }^{86}$ Copyright 2015, Royal Society of Chemistry. 
Electrowetting based control of LM in capillaries is similar in principle to CEW. By applying a positive potential to the electrolyte relative to the LM, a surface tension gradient is generated on the $\mathrm{LM}^{59}$ For the case of $\mathrm{LM}$ in a reservoir connected to a channel, the point of the LM with the lowest interfacial tension is at the entrance to the channel. The interfacial tension gradient results in Marangoni flow along the channel, which acts as a conveyor belt to pull the LM out of the reservoir. If this force is greater than the capillary pressure, the $\mathrm{LM}$ is pulled into the channel. ${ }^{59}$ Small capillary sidechannels can be used in addition to the main channel to ensure LM does not block the flow of electrolyte.

Alternatively, applying a negative potential to the electrolyte relative to the LM causes oxidation of the LM. ${ }^{59}$ This also causes LM to move along the channel from the reservoir due to interfacial tension reduction and Marangoni flow. The potential used dictates how far the LM will travel along the channel, as when the LM gets closer to the negative electrode and the resistance decreases, the current increases, which leads to more rapid oxide growth. This can eventually stop the LM actuation. The oxide growth has been used to make LM retain its shape in a channel after actuating it to a desired position (Fig. 4D). ${ }^{59}$ Reducing the oxide will then quickly bring the LM back to the reservoir.

Electrocapillarity is also able to steer LM as it is pumped along branching microchannels. ${ }^{86}$ Applying a positive potential to an electrolyte relative to LM in one of two branching channels causes LM to flow preferentially to the channel with the applied potential, as that requires less energy (Fig. 4E). Alternatively, applying a high $(\sim 5 \mathrm{~V})$ oxidative potential to the LM in electrolyte causes a thick oxide layer to form as it nears the negative electrode. This blocks the channel, making the LM travel along the other channel. ${ }^{86}$

LM has been used in reconfigurable antennas that use electrocapillary actuation. ${ }^{87,88}$ One antenna design varied its polarization by making LM move into 5 discrete states by filling different channels that were connected to a central reservoir. There were also notches at the end of the channels to create a minimum energy state for the LM, keeping it in position without having to use any power. ${ }^{87}$ Another design combined electrocapillarity and CEW of LM in a reconfigurable LM pixel array. $^{88}$

\subsection{Electrowetting on dielectric (EWOD)}

Electrowetting on dielectric is a widely used technique to modify the surface wetting properties of conductive liquid droplets. $^{89}$ A droplet placed on a dielectric surface, with an electrode beneath it and an electric potential applied, causes a redistribution of charges towards the droplet-surface interface. The charge accumulation on the surface of the droplet reduces its surface tension and hence its wetting angle. Surface tension varies according to the Young-Lippman equation, which here is written to show how the angle changes based on the applied voltage:

$$
\cos \theta=\cos \theta_{\gamma}-\frac{\varepsilon_{\mathrm{r}} \varepsilon_{0}}{2 d \gamma_{\mathrm{LV}}}\left(V-V_{0}\right)^{2}
$$

where $\theta$ is the contact angle, $\theta_{\gamma}$ is the Young's contact angle under zero applied potential, $\varepsilon$ is the relative permittivity of the dielectric used, $\varepsilon_{0}$ is the permittivity of free space, $d$ is the dielectric layer thickness, and $\gamma_{\mathrm{LV}}$ is the interfacial tension between the liquid and vapor phases. ${ }^{89}$

Using LM for EWOD has some challenges associated with it. For example, the oxide layer adheres well to most surfaces and can impede the actuation of the LM. One solution is to prevent the oxide layer forming by reducing the oxygen concentration $<1$ ppm. ${ }^{28}$ However, this is not feasible for most applications. Alternatively, infiltrating a silicone oil with $\mathrm{HCl}$ results in an acidic oil that is electrically insulating and removes the oxide layer. ${ }^{90}$ Also, the very high surface tension of LM means that a high voltage $(>1 \mathrm{kV})$ is required to be able to change the surface tension appreciably. This high voltage, coupled with the thin dielectric layer means it nears or is greater than the dielectric breakdown limit for some materials. To reduce the voltage required, the shape of the channel can be changed so that the LM droplet is actuated with a lower electrostatic pressure. This technique was applied to actuate LM in acidic silicone oil using EWOD, with grooved channels in order to reduce the difference in electrostatic pressure required to merge the droplets (Fig. 5A). ${ }^{90}$ The shape of the grooves also
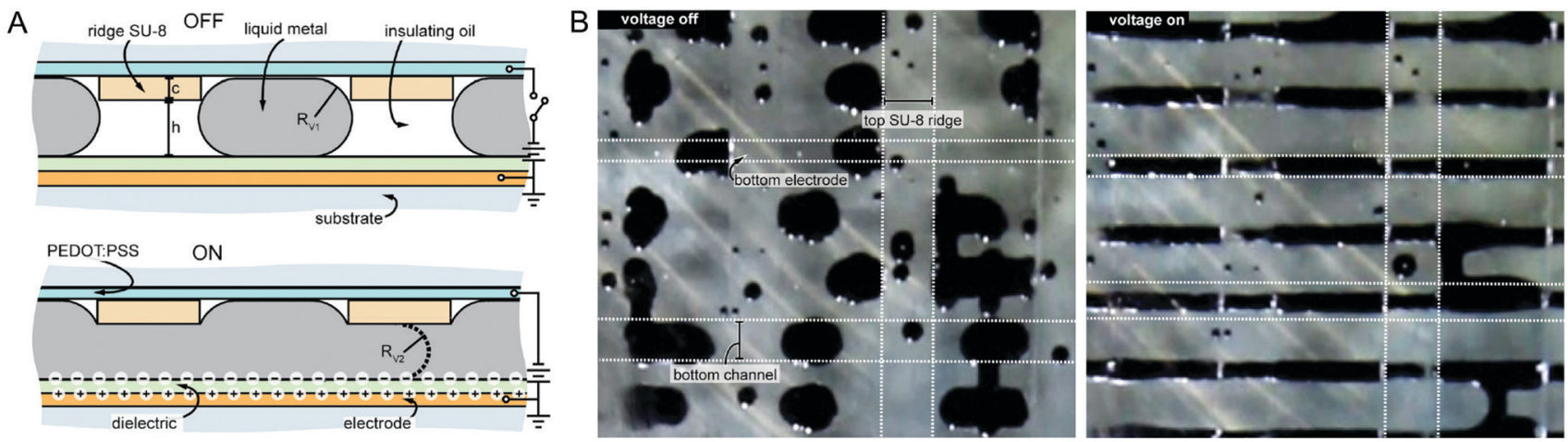

Fig. 5 Actuators enabled by EWOD. (A) Schematic of the EWOD LM polarizer. The ridges cause the pressure differential between the two states to be reduced and break the LM apart after the voltage is removed. (B) Picture of the LM polarizer with voltage off and voltage on. Reproduced with permission. ${ }^{90}$ Copyright 2017, IOP. 

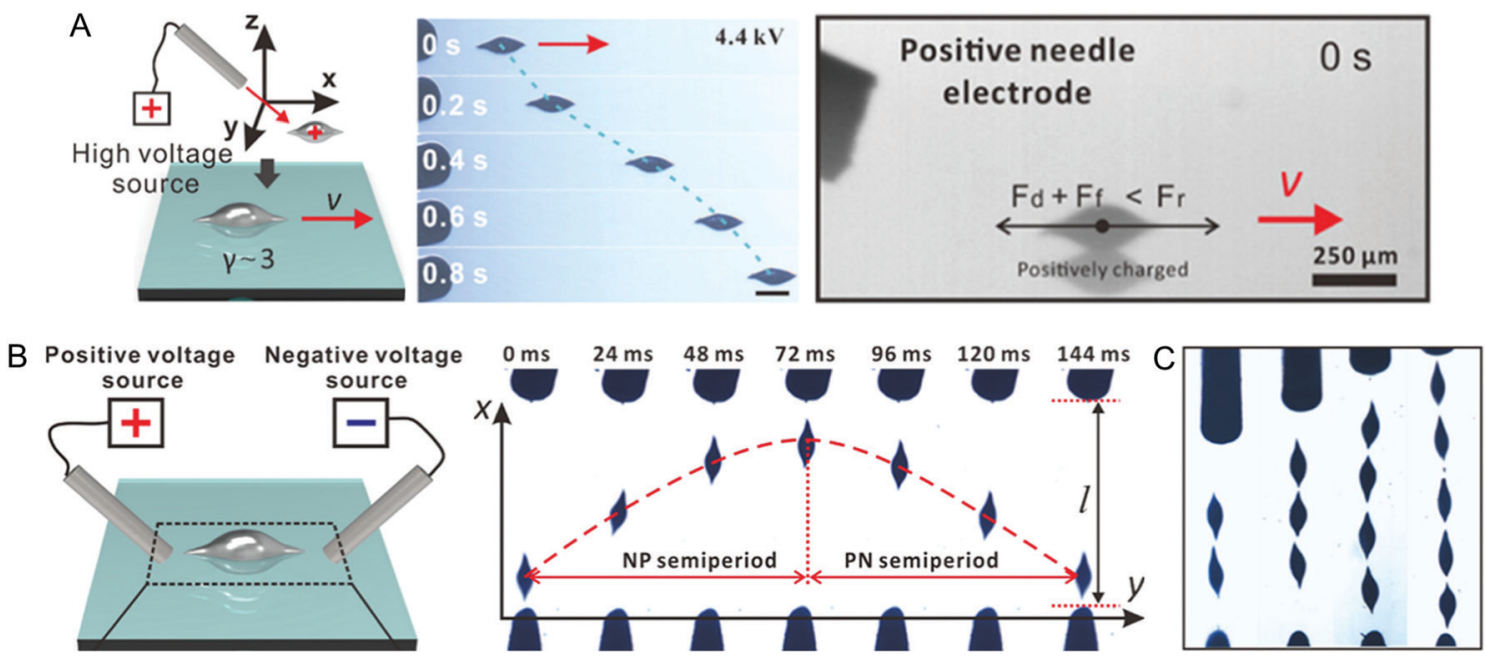

Fig. 6 Electrostatic actuation of LM. (A) Repelling a LM droplet from a high voltage probe. (B) Oscillating movement of LM between two probes. (C) LM droplets lining up along the electric field. Reproduced with permission. ${ }^{91}$ Copyright 2021, ACS.

meant the droplets separated when the voltage was removed (Fig. 5B). The LM EWOD device was used as an electromagnetic polarizer with a low switching time of $\sim 12 \mathrm{~ms}$.

\subsection{Electrostatic}

Electrostatic forces have also been used to successfully actuate non-spherical LM particles electrophoretically. ${ }^{91}$ The particles were created using shear forces to pinch off LM flow out of a capillary tube. The silicone oil used as the fluid was saturated with oxygen to ensure rapid oxidation of the LM. The oxide layer stabilised the non-spherical shape formed by the shearing forces. Various morphologies were created, such as an ellipsoid, single tail, double tail and rod, and had a size of $\sim 250 \mu \mathrm{m}$. The particles were actuated using a high potential difference

A
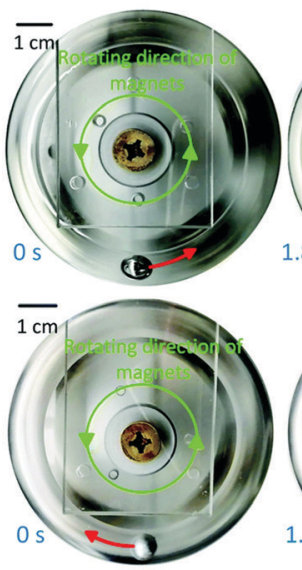

1.85
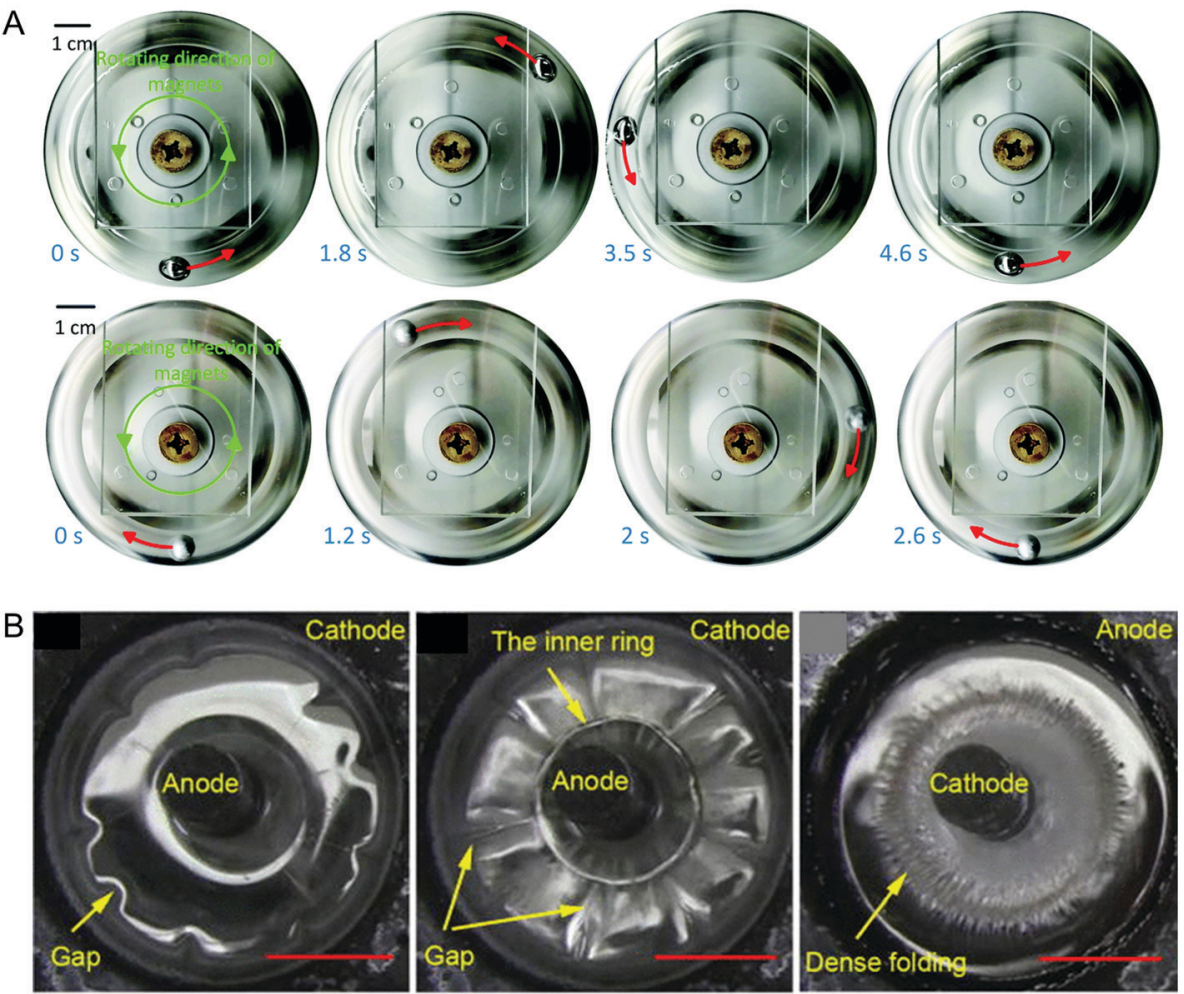

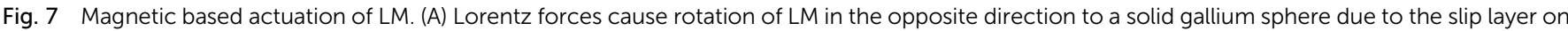

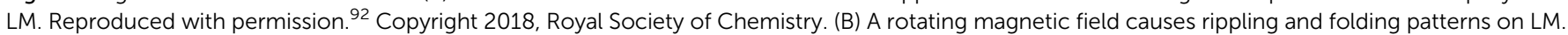
Reproduced with permission. ${ }^{93}$ Copyright 2016, American Institute of Physics. 
Table 1 Details of the different LM actuation types

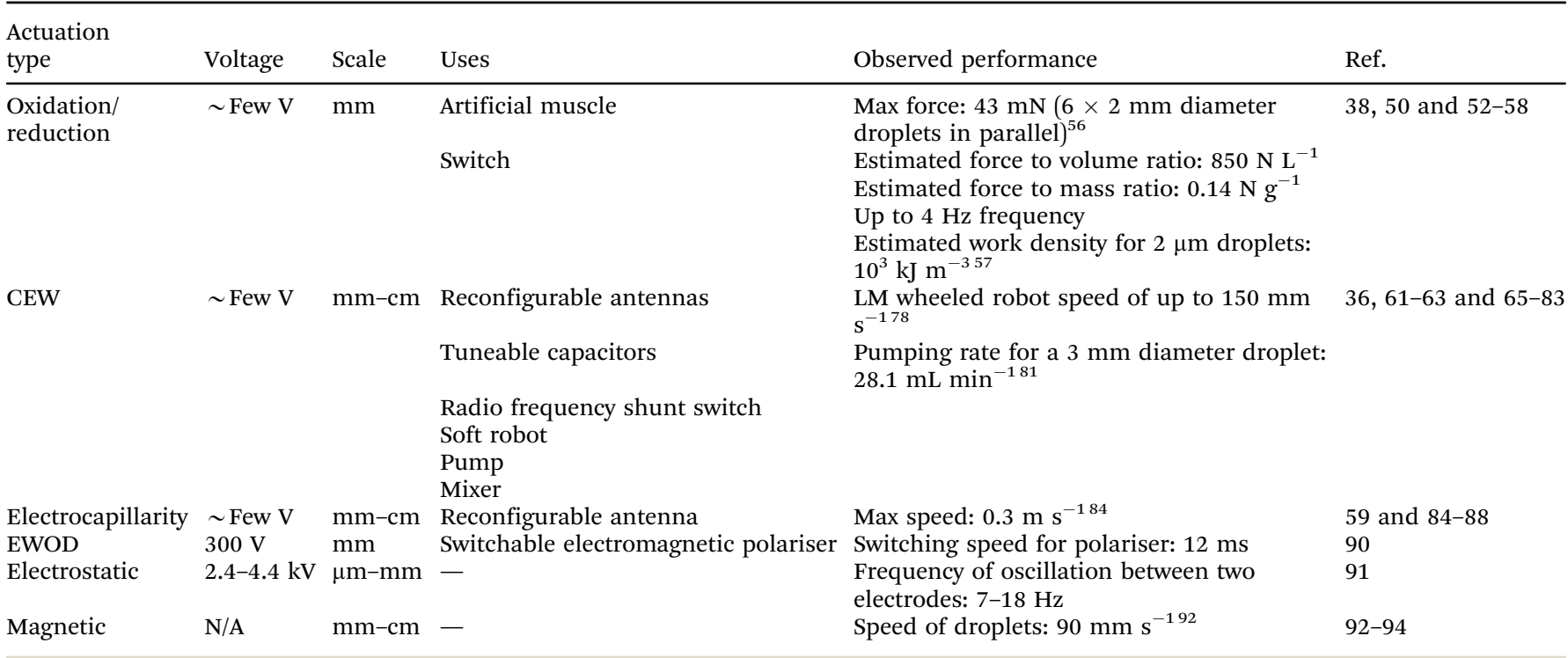

$(\sim$ few $\mathrm{kV})$ with needle electrodes. Bringing a charged electrode towards a particle and contacting it caused it to be charged. It then was repelled due to electrostatic forces (Fig. 6A). Placing the positive and the negative electrode opposite each other made the particle move between the electrodes. At first the LM particle is positively charged and moves towards the negative electrode, then it becomes negatively charged and moves the other way (Fig. 6B). The period of the oscillation reduces with increasing potential difference between the electrodes, from a $148 \mathrm{~ms}$ period at $2.4 \mathrm{kV}$ potential difference, to $55 \mathrm{~ms}$ at $3.6 \mathrm{kV}$. The thinner tails of the particles line up with the applied electric field due to uneven charge distribution. Multiple particles line up in series between the two electrodes (Fig. 6C), and can form a short-lived electrical connection, which is destroyed as a result of the high voltage causing rapid electrolysis.

\subsection{Magnetic}

The high conductivity of LMs means that a changing magnetic field is able to induce a large enough eddy current for Lorentz forces to move a $\mathrm{LM}$ droplet in $\mathrm{NaOH}$ solution. ${ }^{92}$ The alkaline solution is required to remove the oxide layer to prevent the LM droplet sticking to the container. Interestingly, the LM droplet moves in the opposite direction to solid gallium and copper spheres under the same conditions (Fig. 7A). The Lorentz forces experienced by solid metal spheres result in a horizontal force (which is in the same direction as the moving magnet), and a torque that makes it rotate towards the opposite direction. The slip layer on LM acts as a lubricant, preventing it rolling. Therefore, LM moves in the opposite direction to solid spheres, which roll due to the applied torque.

Rotating magnetic fields have been shown to induce surface patterns in large LM droplets (Fig. 7B). ${ }^{93}$ The setup used comprises a LM droplet in $\mathrm{NaOH}$ electrolyte, with graphite electrodes in the centre and around the outside. With a rotating magnetic field and a low potential applied $(2.5 \mathrm{~V})$, the LM rotates around the inner electrode due to Lorentz forces. With an increased potential $(4 \mathrm{~V})$, the LM stops rotating, and displays the folding and rippling patterns. The combination of the applied potential and the rotating magnetic field causes variations in interfacial tension to generate the patterns.

A ferromagnetic LM alloy has been made that is able to be actuated electrically by CEW and using magnetic fields. ${ }^{94} \mathrm{LM}$ was mixed with copper-iron nanoparticles in $\mathrm{HCl}$ solution to create the ferromagnetic LM. The $\mathrm{HCl}$ solution removed the oxide on the LM and on the nanoparticles. A galvanic cell is also formed between the $\mathrm{Ga}$ and the copper-iron nanoparticles in $\mathrm{HCl}$ solution, oxidising the $\mathrm{Ga}$ and preventing the nanoparticles from dissolving in the $\mathrm{HCl}$ solution. Mixing for 30 minutes resulted in the LM gaining a thick solid shell composed of various oxides and alloys. CEW could be used to separate the more fluidic LM core from the solid shell. The resultant fluidic, ferromagnetic LM had a greater weight content of copper-iron with larger amount of nanoparticle added initially. LM alloys with a higher weight content of copper-iron had increased viscosity, which resulted in slower actuation speed during CEW and a reduced pumping flow rate, but had greater magnetic sensitivity.

\section{Conclusion and outlook}

In this review, the electrical methods to actuate LMs have been discussed, along with the many different applications for this, such as in reconfigurable antennas, pumps, switches, motors, and artificial muscles (Table 1). It is certain that the unique and beneficial properties of LM means that it offers great advantages to a range of technologies. However, there are still some challenges that must be overcome in order for LM enabled electromechanical actuator-based devices to become widely accepted and even commercially viable. 
For example, electrolyte is needed for most of the LM actuation types. This liquid-liquid system has to be carefully sealed, and is difficult to get useful work out of. Electrolyte is especially unwanted in radio frequency devices, where it introduces additional losses. Moreover, electrochemical oxidation/reduction and CEW effects for GaLMs work best in a strong basic solution or acidified KI solution; this compromises the performance and limits the applications of LM actuators in neutral, other acidic, and non-ionic liquids. Instead of using electrolyte and electrical based actuation, pressure can be used in conjunction with a non-wetting coating. However, this introduces additional components and doesn't use one of the benefits of LM (low voltage electrical actuation). An acidic silicone-based oil has been shown to remove the oxide layer for EWOD, however this is a corrosive liquid and is electrically insulating so cannot be used for CEW or oxidation/ reduction.

Electrolysis is another issue. Bubbles of hydrogen are generated at the cathode during electrochemical reactions in aqueous solutions when the potential difference used is $>1.2 \mathrm{~V}$. The bubble generation can impact the performance of the LM actuator itself (for example by blocking the actuation of LM artificial muscle). Electrolysis also reduces the efficiency of the actuation. Using an ionic liquid rather than a $\mathrm{NaOH}$ solution is one potential remedy, as LM has been shown to be able to pump an ionic liquid. Finding an ionic liquid that is safe to use, doesn't generate gas bubbles at the potential voltages needed and able to be used for oxidation/reduction and CEW would be of great benefit.

Another challenge is how best to induce larger forces with LMs? The actuation methods mentioned in this review mainly use interfacial tension modulation to induce motion. The artificial muscles created were only able to lift $\sim 1 \mathrm{~g}$, whereas for most real-world applications the forces required will be $>100$ times greater. Decreasing the size of LM droplets would increase the surface area to volume ratio, which scales with the inverse of the droplet radius. This would result in actuators with a greater power density. However, this could also be more complex to make and to get work out of the system.

\section{Author contributions}

Tim Cole: conceptualisation, writing - original draft. Shi-Yang Tang: conceptualisation, supervision, writing - review \& editing.

\section{Conflicts of interest}

There are no conflicts to declare.

\section{Acknowledgements}

S.-Y.T. is grateful for the support from the Royal Society, UK (IEC/NSFC/201223).

\section{References}

1 N. El-Atab, R. B. Mishra, F. Al-Modaf, L. Joharji, A. A. Alsharif, H. Alamoudi, M. Diaz, N. Qaiser and M. M. Hussain, Adv. Intell. Syst., 2020, 2, 2000128.

2 L. Hines, K. Petersen, G. Z. Lum and M. Sitti, Adv. Mater., 2017, 29, 1603483.

3 P. Rothemund, N. Kellaris, S. K. Mitchell, E. Acome and C. Keplinger, Adv. Mater., 2021, 33, e2003375.

4 M. Duduta, E. Hajiesmaili, H. Zhao, R. J. Wood and D. R. Clarke, Proc. Natl. Acad. Sci. U. S. A., 2019, 116, 2476-2481.

5 V. Cacucciolo, J. Shintake, Y. Kuwajima, S. Maeda, D. Floreano and H. Shea, Nature, 2019, 572, 516-519.

6 Y. Kim, G. A. Parada, S. Liu and X. Zhao, Sci. Robot., 2019, 4, 1-15.

7 W. Hu, G. Z. Lum, M. Mastrangeli and M. Sitti, Nature, 2018, 554, 81-85.

8 S. Y. Tang, X. C. Zhang, S. S. Sun, D. Yuan, Q. B. Zhao, S. Yan, L. Deng, G. L. Yun, J. Zhang, S. W. Zhang and W. H. Li, Adv. Funct. Mater., 2018, 28, 1705484.

9 Y. Tang, Y. Chi, J. Sun, T. H. Huang, O. H. Maghsoudi, A. Spence, J. Zhao, H. Su and J. Yin, Sci. Adv., 2020, 6, eaaz6912.

10 J. Walker, T. Zidek, C. Harbel, S. Yoon, F. S. Strickland, S. Kumar and M. Shin, Actuators, 2020, 9, 3.

11 H. Kim, S. K. Ahn, D. M. Mackie, J. Kwon, S. H. Kim, C. Choi, Y. H. Moon, H. B. Lee and S. H. Ko, Mater. Today, 2020, 41, 243-269.

12 H. Lu, G. Yun, T. Cole, Y. Ouyang, H. Ren, J. Shu, Y. Zhang, S. Zhang, M. D. Dickey, W. Li and S. Y. Tang, ACS Appl. Mater. Interfaces, 2021, 13, 37904-37914.

13 H. K. Bisoyi and Q. Li, Chem. Rev., 2016, 116, 15089-15166. 14 Y. Liu, B. Shaw, M. D. Dickey and J. Genzer, Sci. Adv., 2017, 3, e1602417.

15 S. J. Park, M. Gazzola, K. S. Park, S. Park, V. Di Santo, E. L. Blevins, J. U. Lind, P. H. Campbell, S. Dauth, A. K. Capulli, F. S. Pasqualini, S. Ahn, A. Cho, H. Yuan, B. M. Maoz, R. Vijaykumar, J. W. Choi, K. Deisseroth, G. V. Lauder, L. Mahadevan and K. K. Parker, Science, 2016, 353, 158-162.

16 N. W. Bartlett, M. T. Tolley, J. T. Overvelde, J. C. Weaver, B. Mosadegh, K. Bertoldi, G. M. Whitesides and R. J. Wood, Science, 2015, 349, 161-165.

17 R. F. Shepherd, A. A. Stokes, J. Freake, J. Barber, P. W. Snyder, A. D. Mazzeo, L. Cademartiri, S. A. Morin and G. M. Whitesides, Angew. Chem., Int. Ed., 2013, 52, 2892-2896.

18 A. Kotikian, J. M. Morales, A. Lu, J. Mueller, Z. S. Davidson, J. W. Boley and J. A. Lewis, Adv. Mater., 2021, 33, e2101814.

19 M. J. Ford, M. Palaniswamy, C. P. Ambulo, T. H. Ware and C. Majidi, Soft Matter, 2020, 16, 5878-5885.

20 C. Pan, E. J. Markvicka, M. H. Malakooti, J. Yan, L. Hu, K. Matyjaszewski and C. Majidi, Adv. Mater., 2019, 31, e1900663.

21 M. Y. Hao, Y. J. Wang, Z. C. Zhu, Q. S. He, D. L. Zhu and M. Z. Luo, Front. Robot. AI, 2019, 6, 129. 
22 J. D. Carrico, T. Hermans, K. J. Kim and K. K. Leang, Sci. Rep., 2019, 9, 17482.

23 D. Morales, E. Palleau, M. D. Dickey and O. D. Velev, Soft Matter, 2014, 10, 1337-1348.

24 T. Nagaoka, Z. B. Mao, K. Takemura, S. Yokota and J. W. Kim, Smart Mater. Struct., 2019, 28, 025032.

25 J. Kedzierski, K. Meng, T. Thorsen, R. Cabrera and S. Berry, J. Microelectromech. Syst., 2016, 25, 394-400.

26 J. Kedzierski and H. Chea, Microsyst. Nanoeng., 2021, 7, 22.

27 A. Tonazzini, A. Sadeghi and B. Mazzolai, Soft Robot., 2016, 3, 34-41.

28 T. Liu, P. Sen and C.-J. Kim, J. Microelectromech. Syst., 2012, 21, 443-450.

29 M. D. Dickey, R. C. Chiechi, R. J. Larsen, E. A. Weiss, D. A. Weitz and G. M. Whitesides, Adv. Funct. Mater., 2008, 18, 1097-1104.

30 Y. Lin, J. Genzer and M. D. Dickey, Adv. Sci., 2020, 7, 2000192.

31 X. Wang, L. Fan, J. Zhang, X. Sun, H. Chang, B. Yuan, R. Guo, M. Duan and J. Liu, Adv. Funct. Mater., 2019, 29, 1907063.

32 M. D. Dickey, ACS Appl. Mater. Interfaces, 2014, 6, 18369-18379.

33 D. Zrnic and D. S. Swatik, J. Less-Common Met., 1969, 18, 67-68.

34 S. Handschuh-Wang, Y. Chen, L. Zhu and X. Zhou, ChemPhysChem, 2018, 19, 1584-1592.

35 S. Handschuh-Wang, T. Gan, T. Wang, F. J. Stadler and X. Zhou, Langmuir, 2021, 37, 9017-9025.

36 S. Handschuh-Wang, Y. Chen, L. Zhu, T. Gan and X. Zhou, Langmuir, 2019, 35, 372-381.

37 Y. Chung and C.-W. Lee, J. Electrochem. Sci. Technol., 2013, 4, 1-18.

38 M. R. Khan, C. B. Eaker, E. F. Bowden and M. D. Dickey, Proc. Natl. Acad. Sci. U. S. A., 2014, 111, 14047-14051.

39 B. A. Gozen, A. Tabatabai, O. B. Ozdoganlar and C. Majidi, Adv. Mater., 2014, 26, 5211-5216.

40 J. Ma, V. T. Bharambe, K. A. Persson, A. L. Bachmann, I. D. Joshipura, J. Kim, K. H. Oh, J. F. Patrick, J. J. Adams and M. D. Dickey, ACS Appl. Mater. Interfaces, 2021, 13, 12709-12718.

41 K. B. Ozutemiz, J. Wissman, O. B. Ozdoganlar and C. Majidi, Adv. Mater. Interfaces, 2018, 5, 1701596.

42 Y.-G. Deng and J. Liu, Appl. Phys. A: Mater. Sci. Process., 2009, 95, 907-915.

43 S. Handschuh-Wang, F. J. Stadler and X. C. Zhou, J. Phys. Chem. C, 2021, 125, 20113-20142.

44 S. Y. Tang, C. Tabor, K. Kalantar-Zadeh and M. D. Dickey, Annual Review of Materials Research, 2021, vol. 51, pp. 381408.

45 T. Daeneke, K. Khoshmanesh, N. Mahmood, I. A. de Castro, D. Esrafilzadeh, S. J. Barrow, M. D. Dickey and K. KalantarZadeh, Chem. Soc. Rev., 2018, 47, 4073-4111.

46 J. Zhang, Y. Yao, L. Sheng and J. Liu, Adv. Mater., 2015, 27, 2648-2655.

47 S. Xu, B. Yuan, Y. Hou, T. Y. Liu, J. H. Fu and J. Liu, J. Phys. D: Appl. Phys., 2019, 52, 353002.
48 X. Wang, R. Guo and J. Liu, Adv. Mater. Technol., 2018, 4, 1800549.

49 C. B. Eaker and M. D. Dickey, Appl. Phys. Rev., 2016, 3, 031103.

50 C. B. Eaker, D. C. Hight, J. D. O'Regan, M. D. Dickey and K. E. Daniels, Phys. Rev. Lett., 2017, 119, 174502.

51 M. Y. Song, K. E. Daniels, A. Kiani, S. Rashid-Nadimi and M. D. Dickey, Adv. Intell. Syst., 2021, 3, 2100024.

52 M. Y. Song, N. Mehrabian, S. Karuturi and M. D. Dickey, Appl. Phys. Lett., 2021, 118, 081601.

53 Z. Yu, Y. Chen, F. F. Yun, D. Cortie, L. Jiang and X. Wang, Phys. Rev. Lett., 2018, 121, 024302.

54 M. Li, H. Mohamed Cassim Mohamed Anver, Y. Zhang, S. Y. Tang and W. Li, Micromachines, 2019, 10, 209.

55 J. Wissman, M. D. Dickey and C. Majidi, Adv. Sci., 2017, 4, 1700169.

56 J. Shu, D. A. Ge, E. Wang, H. Ren, T. Cole, S. Y. Tang, X. Li, X. Zhou, R. Li, H. Jin, W. Li, M. D. Dickey and S. Zhang, Adv. Mater., 2021, 33, e2103062.

57 J. Liao and C. Majidi, Soft Matter, 2021, 17, 1921-1928.

58 L. Russell, J. Wissman and C. Majidi, Appl. Phys. Lett., 2017, 111, 254101.

59 R. C. Gough, A. M. Morishita, J. H. Dang, M. R. Moorefield, W. A. Shiroma and A. T. Ohta, Micro Nano Syst. Lett., 2015, 3,4 .

60 L. Junghoon and K. Chang-Jin, J. Microelectromech. Syst., 2000, 9, 171-180.

61 S. J. Dacuycuy, W. A. Shiroma and A. T. Ohta, presented in part at the 2021 20th IEEE Intersociety Conference on Thermal and Thermomechanical Phenomena in Electronic Systems (iTherm), 2021.

62 K. J. Sarabia, S. S. Yamada, R. C. Gough, M. R. Moorefield, A. W. Combs, W. A. Shiroma and A. T. Ohta, Electron. Lett., 2017, 53, 1635-1636.

63 H. Ren, H. Jin, J. Shu, J. Xie, E. Wang, D. A. Ge, S. Y. Tang, X. Li, W. Li and S. Zhang, Mater. Horiz., 2021, 8, 3063-3071.

64 M. Kosmulski, Chemical properties of material surfaces, CRC press, 2001.

65 S. Y. Tang, V. Sivan, K. Khoshmanesh, A. P. O’Mullane, X. Tang, B. Gol, N. Eshtiaghi, F. Lieder, P. Petersen, A. Mitchell and K. Kalantar-zadeh, Nanoscale, 2013, 5, 5949-5957.

66 J. Ye, S. C. Tan, L. Wang and J. Liu, Soft Matter, 2021, 17, 7835-7843.

67 V. Sivan, S. Y. Tang, A. P. O'Mullane, P. Petersen, N. Eshtiaghi, K. Kalantar-zadeh and A. Mitchell, Adv. Funct. Mater., 2013, 23, 144-152.

68 V. Sivan, S.-Y. Tang, A. P. O’Mullane, P. Petersen, K. Kalantar-zadeh, K. Khoshmanesh and A. Mitchell, Appl. Phys. Lett., 2014, 105, 121607.

69 R. C. Gough, A. M. Morishita, J. H. Dang, W. Q. Hu, W. A. Shiroma and A. T. Ohta, IEEE Access, 2014, 2, 874-882.

70 M. W. Sahara, J. A. Marutani, A. T. Ohta and W. A. Shiroma, Presented in part at the 2021 IEEE Texas Symposium on Wireless and Microwave Circuits and Systems (WMCS), 2021. 
71 Y. Y. Yao and J. Liu, $R S C A d v .$, 2016, 6, 56482-56488.

72 J. Wu, S. Y. Tang, T. Fang, W. Li, X. Li and S. Zhang, Adv. Mater., 2018, 30, e1805039.

73 R. Xue, Y. Tao, H. Sun, W. Liu, Z. Ge, T. Jiang, H. Jiang, F. Han, Y. Li and Y. Ren, Lab Chip, 2021, 21, 2771-2780.

74 S. Y. Tang, K. Khoshmanesh, V. Sivan, P. Petersen, A. P. O'Mullane, D. Abbott, A. Mitchell and K. KalantarZadeh, Proc. Natl. Acad. Sci. U. S. A., 2014, 111, 3304-3309.

75 S. Y. Tang, V. Sivan, P. Petersen, W. Zhang, P. D. Morrison, K. Kalantar-zadeh, A. Mitchell and K. Khoshmanesh, Adv. Funct. Mater., 2014, 24, 5851-5858.

76 G. B. Zhang, R. C. Gough, M. R. Moorefield, K. S. Elassy, A. T. Ohta and W. A. Shiroma, Int. J. Antennas Propag., 2018, 2018, 1-7.

77 M. R. Moorefield, R. C. Gough, A. T. Ohta and W. A. Shiroma, IEEE Access, 2018, 6, 1261-1266.

78 X. X. Li, J. Xie, S. Y. Tang, R. Xu, X. P. Li, W. H. Li and S. W. Zhang, IEEE Trans. Ind. Informat., 2019, 15, 2535-2543.

79 F. X. Li, J. Shu, L. R. Zhang, N. L. Yang, J. Xie, X. P. Li, L. Cheng, S. L. Kuang, S. Y. Tang, S. W. Zhang, W. H. Li, L. N. Sun and D. Sun, Appl. Mater. Today, 2020, 19, 100597. 80 E. Wang, J. Shu, H. Jin, Z. Tao, J. Xie, S. Y. Tang, X. Li, W. Li, M. D. Dickey and S. Zhang, iScience, 2021, 24, 101911.

81 J. Y. Zhu, S. Y. Tang, K. Khoshmanesh and K. Ghorbani, ACS Appl. Mater. Interfaces, 2016, 8, 2173-2180.

82 R. Xue, W. Liu, T. Jiang, C. Song, H. Jiang and Y. Ren, Adv. Mater. Interfaces, 2020, 7, 2000345.
83 Q. M. Hu, Y. K. Ren, W. Y. Liu, X. M. Chen, Y. Tao and H. Y. Jiang, Micromachines, 2017, 8, 119.

84 M. R. Khan, C. Trlica and M. D. Dickey, Adv. Funct. Mater., 2015, 25, 671-678.

85 M. R. Khan, J. Bell and M. D. Dickey, Adv. Mater. Interfaces, 2016, 3, 1600546.

86 S. Y. Tang, Y. Lin, I. D. Joshipura, K. Khoshmanesh and M. D. Dickey, Lab Chip, 2015, 15, 3905-3911.

87 G. B. Zhang, R. C. Gough, M. R. Moorefield, K. J. Cho, A. T. Ohta and W. A. Shiroma, IEEE Antennas Wireless Propag. Lett., 2018, 17, 50-53.

88 K. S. Elassy, K. J. Sarabia, W. A. Shiroma and A. T. Ohta, Presented in part at the 2021 IEEE 21st Annual Wireless and Microwave Technology Conference (WAMICON), 2021.

89 F. Mugele and J. C. Baret, J. Phys.: Condens. Matter, 2005, 17, R705-R774.

90 A. V. Diebold, A. M. Watson, S. Holcomb, C. Tabor, D. Mast, M. D. Dickey and J. Heikenfeld, J. Micromech. Microeng., 2017, 27, 025010.

91 S. Wang, Z. Zhu, C. Ma, R. Qiao, C. Yang, R. X. Xu and T. Si, ACS Appl. Mater. Interfaces, 2021, 13, 16677-16687.

92 J. Shu, S. Y. Tang, Z. Feng, W. Li, X. Li and S. Zhang, Soft Matter, 2018, 14, 7113-7118.

93 L. Wang and J. Liu, Appl. Phys. Lett., 2016, 108, 161602.

94 F. X. Li, S. L. Kuang, X. P. Li, J. Shu, W. H. Li, S. Y. Tang and S. W. Zhang, Adv. Mater. Technol., 2019, 4, 1800694. 